\title{
PERCEPÇÃO DOS PROFISSIONAIS DE SAÚDE DA ATENÇÃO BÁSICA SOBRE OS GRUPOS DE GESTANTES
}

\author{
PERCEPTION OF ATTENTION HEALTH PROFESSIONALS \\ ON BASIC GROUPS OF PREGNANT WOMEN
}

\section{Joice Ane Teixeira', Marilu Correa Soares', Ana Paula de Lima Escobal', Kamila Dias Gonçalves', Greice Carvalho de Matos', Bruna Madruga Pires da Silva', Katia da Silva Rocha²}

\section{RESUMO}

Objetivo: Conhecer a percepção dos profissionais de saúde da Atenção Básica sobre os grupos de gestantes na atenção ao pré-natal, parto e puerpério. Metodologia: estudo de abordagem qualitativa descritiva, realizado em duas Unidades Básicas de Saúde de uma cidade do Sul do Brasil, nos meses de abril e maio de 2015. Os participantes do estudo foram 13 profissionais graduados da equipe de saúde. Os dados foram submetidos à análise temática. Resultados: o estudo apontou que os profissionais de saúde foram unânimes em reconhecer o trabalho com grupos de gestantes como uma atividade relevante e proveitosa e referiram que não tiveram suporte teórico-prático durante a formação acadêmica para o desenvolvimento da prática educativa com grupos. Considerações finais: o trabalho com grupos de gestantes foi reconhecido pelos profissionais de saúde como importante ferramenta na atenção ao pré-natal, parto e puerpério.

Descritores: Atenção Primária a Saúde; Gestantes; Saúde da Mulher; Promoção da saúde.

\begin{abstract}
Objective: To know the primary health care professionals' perceptions about the groups of pregnant women under attention to prenatal care, parturition and puerperium. Methodology: it is a qualitative and descriptive study, which was carried out in two primary health care units in a city in the south of Brazil, in April and May 2015. Participantswere 13 graduatedprofessionalsinserted in thehealthteam. Data was submitted to thematic analysis. Results: the study pointed out that health professionals were unanimousto recognize that the groups for pregnant women are a relevant and profitable activity, referring that they had no theoretical/practical support during academic education to develop educational practices with groups. Final Considerations: working with groups of pregnant women was recognized by health professionals as an important tool in attention to prenatal, parturition and postpartum care.
\end{abstract}

Descriptors: Primary Health Care; Pregnant Women; Women's Health; Health Promotion. 


\section{Introdução}

A gestação é um período permeado por grandes mudanças. 0 início e o desenvolvimento de uma gravidez geralmente são percebidos como fenômenos complexos, sendo marcados por profundas modificações fisiológicas, psicológicas e de âmbito social. Sendo que estas podem interferir no mundo intrapsíquico e relacional da mulher, alterando significativamente a visão que ela tem de si mesma e da sua relação com o mundo'.

As gestantes têm necessidade de expressarem seus sentimentos, serem ouvidas e obterem respostas às suas inquietações. Neste sentido, uma alternativa para o esclarecimento destes questionamentos é o grupo de gestantes. Por ser um espaço no qual a mulher pode expressar e refletir sobre seus problemas e angústias, assim como trocar experiências².

As atividades em grupo aumentam a possibilidade de relações mais solidárias, uma vez que as pessoas se identificam em suas semelhanças, e produzem ideias e ações coletivamente. Além disso, esta atividade propicia a interação entre os participantes, enriquecendo a qualidade do conhecimento e a aquisição de novos saberes ${ }^{3}$.

Em consonância, um estudo realizado no sul do Rio Grande do Sul sobre as potencialidades e/ou fragilidades do grupo de gestantes como espaço de trocas de saberes e práticas, evidenciou que o grupo foi um espaço no qual as mulheres mostraram-se mais aptas e autônomas para 0 autocuidado e o cuidado com o recém-nascido. A metodologia grupal também proporcionou troca de conhecimentos e experiências 4 .

As práticas educativas propiciam por meio das atividades a troca de conhecimento entre os participantes dos grupos, sendo desta forma considerada uma atenção diferenciada nos serviços de saúde. À medida que os profissionais permitem a atuação dos usuários, o processo comunicativo melhora, resultando em troca e compartilhamento de saberes 5 .

No cenário de atenção à mulher que vivencia o processo de gestar e parir, a importância de atividades com grupos está ancorada na possibilidade de fornecer um processo de parturição mais tranqüilo às gestantes. Na perspectiva de abandonarem a postura submissa na sala de parto, tornado-as capazes de tomar as decisões nesse momento, bem como ao longo da assistência pré-natal ofertada 6 .

Nesta conjuntura, entende-se que os profissionais de saúde precisam estar motivados e preparados para trabalhar com a metodologia grupal, sendo facilitadores da interação entre os membros do grupo, com consciência dos limites e potencialidades de cada participante, possibilitando a interação dos saberes de forma recíproca, bem como prestar um cuidado humanizado. Diante do exposto, construiu-se este estudo norteado pela seguinte questão: qual a percepção dos profissionais de saúde da Atenção Básica sobre os grupos de gestantes na atenção ao pré-natal, parto e puerpério? Para tal, teve-se como objetivo conhecer a percepção dos profissionais de saúde da Atenção Básica sobre os grupos de gestantes na atenção ao pré-natal, parto e puerpério.

\section{Metodologia}

Trata-se de uma pesquisa qualitativa descritiva realizada em duas Unidades Básicas de Saúde (UBSs) de uma cidade do Sul do Estado do Rio Grande do Sul-Brasil. A UBS 1 atende cinco microáreas com cobertura populacional de 3.000 pessoas. A UBS 2 é composta de vinte microáreas e cobertura populacional em torno de 10.800 pessoas.

Os participantes do estudo foram 13 profissionais após ocorrer a saturação teórica ${ }^{7}$, definida como o momento em que pouco de substancialmente novo aparece, levando em consideração cada uma das questões abordadas e/ou identificadas durante a análise. Todos os profissionais eram graduados que faziam parte das equipes de saúde das UBSs no período de coleta de dadosOs critérios de inclusão foram: trabalhar nas UBSs selecionadas por um período mínimo de um ano; ter disponibilidade em participar do estudo; concordar com a divulgação e publicação dos resultados em meios acadêmicos e científicos; permitir o uso de gravador durante as entrevistas. Os critérios de exclusão foram: férias e licença de saúde.

A coleta de dados ocorreu nos meses de abril e maio de 2015, por meio de entrevista semiestruturada. 0 roteiro da entrevista continha questões que investigavam a concepção dos profissionais a cerca do trabalho desenvolvido com grupos de gestantes, bem como o que os mesmos consideravam importante num trabalho com grupos de gestantes, as potencialidades e fragilidades de tal trabalho.

Cabe salientar que as entrevistas foram previamente agendadas e realizadas em uma sala nas dependências das UBS, com duração média de 40 minutos, após, transcritas e analisadas segundo a proposta operativa ${ }^{7}$. 
Os princípios éticos que nortearam esta pesquisa foram os correspondentes a Resolução $n^{0} 466 / 2012$ do Conselho Nacional de Saúde ${ }^{8}$, do Ministério da Saúde, que dispõem sobre Pesquisas com seres humanos e obteve parecer favorável Comitê de Ética em Pesquisa sob o n 971.024. O Termo de Consentimento Livre e Esclarecido (TCLE) foi assinado pelos participantes e 0 anonimato foi garantido pela identificação destes por meio das abreviaturas das profissões acrescidas da ordem numérica das entrevistas. Exemplo: Enfermeiro- Enf1, Enf2; Médico - Med1, Med2; Dentista - Den1, Den2; Assistente Social - AS1, AS2.

\section{Resultados}

Para melhor compreensão dos resultados, apresentaremos os profissionais de saúde que participaram deste estudo, os quais foram identificados pela abreviatura de sua profissão, acompanhado de um número, que representou a ordem de entrevista, seguido da sigla UBS associado a um número que identifica a unidade básica.

Tabela 1 - Apresentação dos Participantes

\begin{tabular}{|c|c|c|c|c|c|c|c|}
\hline Participante & UBS & Profissão & Idade & Estado Civil & $\begin{array}{l}\text { Tempo de } \\
\text { Formado }\end{array}$ & Pós- graduação & $\begin{array}{c}\text { Tempo de } \\
\text { trabalho na UBS }\end{array}$ \\
\hline Enf1 & UBS1 & Enfermeira & 45 & casada & 18anos & -- & 12anos \\
\hline Med 1 & UBS1 & Médica & 37 & divorciada & 17 anos & $\begin{array}{l}\text { Residência } \\
\text { de Medicina } \\
\text { em Saúde e } \\
\text { Comunidade }\end{array}$ & 7 anos \\
\hline AS1 & UBS1 & $\begin{array}{l}\text { Assistente } \\
\text { social }\end{array}$ & 51 & divorciada & 30 anos & $\begin{array}{l}\text { Estratégia da } \\
\text { Saúde da Família, } \\
\text { Políticas Públicas, } \\
\text { Políticas Sociais, } \\
\text { Mestrado e } \\
\text { Doutorado em } \\
\text { Planejamento }\end{array}$ & 11 anos \\
\hline Den 1 & UBS1 & Dentista & 60 & casada & 32 anos & $\begin{array}{l}\text { Mestrado e } \\
\text { Doutorado em } \\
\text { Dentística, }\end{array}$ & 3 anos \\
\hline Enf1 & UBS2 & Enfermeira & 34 & casada & 10anos & $\begin{array}{l}\text { Saúde da Família, } \\
\text { Saúde Pública, } \\
\text { Mestrado em } \\
\text { Ciências da Saúde, }\end{array}$ & $\begin{array}{c}3 \text { anos e } 6 \\
\text { meses }\end{array}$ \\
\hline Enf2 & UBS2 & Enfermeira & 40 & casada & 17 anos & Saúde da Família & 4anos \\
\hline Enf3 & UBS2 & Enfermeiro & 40 & solteiro & 15anos & $\begin{array}{l}\text { Saúde Pública } \\
\text { e Estratégia de } \\
\text { Saúde da Família }\end{array}$ & 11 anos \\
\hline Med 1 & UBS2 & Médica & 57 & divorciada & 33anos & $\begin{array}{l}\text { Estratégia da } \\
\text { Saúde da Família }\end{array}$ & 28 anos \\
\hline Med2 & UBS2 & Médica & 60 & casada & 35anos & $\begin{array}{l}\text { pós-graduação em } \\
\text { Saúde Pública e } \\
\text { em Estratégia de } \\
\text { Saúde da Família } \\
\text { Residência em } \\
\text { Pediatria }\end{array}$ & 10 anos \\
\hline Med3 & UBS2 & Médica & 26 & solteira & $\begin{array}{c}1 \text { ano } \\
6 \text { meses }\end{array}$ & Saúde da Família & 1 ano \\
\hline Med4 & UBS2 & Médica & 35 & casada & 10anos & $\begin{array}{c}\text { Medicina do } \\
\text { Trabalho e Saúde } \\
\text { da Família }\end{array}$ & 2 anos \\
\hline
\end{tabular}




\begin{tabular}{c|c|c|c|c|c|c|c}
\hline Participante & UBS & Profissão & Idade & Estado Civil & $\begin{array}{c}\text { Tempo de } \\
\text { Formado }\end{array}$ & Pós- graduação & $\begin{array}{c}\text { Tempo de } \\
\text { trabalho na UBS }\end{array}$ \\
\hline Med5 & UBS2 & Médica & 58 & divorciada & 34 anos & $\begin{array}{c}\text { Residência } \\
\text { Medicina } \\
\text { Comunitária e } \\
\text { Mestrado Saúde } \\
\text { Coletiva }\end{array}$ & 11 anos \\
\hline AS1 & UBS2 & $\begin{array}{c}\text { Assistente } \\
\text { Social }\end{array}$ & 69 & casada & 37 anos & Saúde da Família & 32 anos \\
\hline
\end{tabular}

Fonte: Tabela elaborada pelos autores da pesquisa.

Os depoimentos dos profissionais de saúde possibilitaram a construção de dois núcleos temáticos. Estes foram denominados como: percepções dos trabalhadores da Atenção Básica sobre o trabalho desenvolvido com grupos de gestantes e Influência do processo de formação dos profissionais de saúde para o trabalho com grupos de gestantes na UBS.

\section{Percepção dos trabalhadores da Atenção Básica sobre o trabalho} desenvolvido com grupos de gestantes

A gravidez pode ser um período de diversas transformações na vida da mulher. A adaptação a esta nova condição pode gerar medo e ansiedade na gestante e em seus familiares. Além das modificações corporais, podem ocorrer mudanças emocionais, promovendo a necessidade de adequação aos novos papéis ${ }^{9}$.

Neste sentido, torna-se interessante proporcionar, durante o pré-natal, espaços para discussões sobre as expectativas relacionadas à maternidade. Como por exemplo, preocupações com a gravidez ou em decorrência dela, como fatores sócio-econômicos, dificuldade de conciliar emprego, abandono dos estudos, entre outras questões ${ }^{10}$.

Frente a estes aspectos, observa-se a importância de um ambiente no qual as gestantes possam expressar suas dúvidas e questionamentos, ter contato com ações educativas, preventivas e promoção de saúde. Neste pensar os grupos de gestantes surgem com tal proposta de trabalho.

Neste sentido os profissionais de saúde participantes deste estudo quando questionados quanto à percepção sobre o trabalho desenvolvido com grupo de gestantes, foram unânimes em reconhecer esta proposta como uma atividade relevante e proveitosa. Fato que é relatado nos depoimentos a seguir.

Eu acho que é muito importante. Eu sempre procurei que o pessoal desenvolvesse aqui, até porque não temos muito tempo. Eu acho importante. Porque junta, agrupa. Elas trocam ideias entre si. E sempre o pessoal aborda os temas que são importantes na gestação. E sempre temos bons resultados do que é trabalhado no grupo. (Enf1 UBS1)

Eu acho tudo válido. Eu quando eu entrei na saúde, eu comecei no posto, ali a gente já fazia grupo de gestantes. (AS1 UBS1)

Ao analisar as falas apresentadas foi possivel inferir que, para os profissionais entrevistados, o grupo de gestante apresentou-se como um dispositivo fundamental junto à equipe de saúde, pois possibilita a troca de informações entre as gestantes e profissionais. Além da troca de saberes, surge como um espaço onde é possível sanar duvidas das gestantes por meio de temas que permeiam a gestação.

Achados semelhantes foram encontrados em uma abordagem realizada com grupos de gestantes, a qual revelou que a participação nestes espaços durante a gestação contribuiu para desmitificar e rever crenças. Além disso, permitiu sanar sentimentos de medo e ansiedade relacionados ao ciclo gravídico-puerperal ${ }^{11}$.

Entende-se que o envolvimento no grupo, possibilita a construção de saberes, estimula a participação do companheiro/acompanhante. Contribuindo para o empoderamento da mulher quanto aos seus direitos, principalmente no processo de parturição.

Nesta perspectiva, as mulheres que participaram de grupos de gestante vivenciaram processos de educação em saúde, compartilharam conhecimentos. Desta forma foi possível realizarem escolhas conscientes sobre suas condutas em relação ao corpo, assim como todas as questões inerentes ao processo do parto ${ }^{4}$. 
No entanto, ainda que os profissionais compreendessem a atividade com grupos como produtiva, observou-se que alguns não vivenciaram esta prática em sua unidade. Verbalizando os motivos pelo qual não desenvolvem grupos de gestantes em suas Unidades Básicas de Saúde.

Aqui na UBS, atualmente, não temos implantado grupo de gestante. Já tivemos em outro momento essa iniciativa, e começamos a fazer o grupo de gestantes. Sempre tivemos essa vontade de fazer. Porém, sempre havia certa dificuldade, uma certa não aceitação das gestantes. (Enf3 UBS2)

Temos muitas falhas ainda. Não temos grupos formados, porque há uma demanda muito grande e acabamos não conseguindo atender todos e termos tempo de fazer os grupos. (Med3 UBS2)

\section{É produtivo, apesar de não conseguirmos manter um grupo grande durante muito tempo. (Med1 UBS2)}

Conforme os relatos acima, observou-se que embora os profissionais reconhecessem a importância do grupo de gestantes não realizam a atividades grupais atualmente. Percebe-se que tal constatação é decorrente de algumas dificuldades como experiências anteriores negativas, excesso de trabalho, baixa adesão das usuárias às atividades propostas, apresentaram-se como entraves para implementação desta atividade.

Em consonância com os resultados desta pesquisa estão um estudo sobre o planejamento das ações educativas pela equipe multiprofissional da Estratégia Saúde da Família cujo tinha o objetivo de identificar como eram planejadas tais ações. Entre as dificuldades encontradas para a realização de práticas educativas estavam a pouca adesão da comunidade, a falta de comprometimento da equipe, o restrito apoio dos gestores, a escassez de recursos financeiros e a falta de espaço físico apropriado ${ }^{12}$.

Em contraponto, autores apontaram que a dificuldade de adesão dos usuários ao grupo estava relacionada à utilização de práticas educativas de abordagem tradicional, ou seja, com temas pré-definidos condicionados aos temas elencados como prioritários pelos serviços de saúde, sem a participação do usuário. Outro obstáculo revelado foi a falta de domínio na coordenação de grupos pelos profissionais ${ }^{13}$.

Quanto à importância da realização de grupo de gestantes, os profissionais relataram aspectos positivos sobre a atividade. Ressaltando as trocas de saberes que foram realizadas entre profissionais de saúde e gestantes, esclarecimento de dúvidas e o incentivo à amamentação.

Eu considero importante, por causa da troca de experiência que elas têm. Porque tu mantendo o grupo de gestante, tem várias etapas, tem gestante de tudo que é idade gestacional, tem gestantes iniciando, tem gestantes multíparas, parto normal, parto cesáreo e ai fica aquela troca intensa de experiências. É muito importante. (Med1 UBS2)

É super importante. Tem muitas que são super inseguras, nunca tiveram filhos e as outras que já tiveram, que estão em outras gestações, podem ajudar, contar como é, incentivar a amamentação, várias coisas assim. (Med3 UBS2)

Porque dúvidas que a gente responde para uma, as outras não tinham, na verdade, não pensaram naquele momento, naquela dúvida e acaba se tornando dúvida de todas. A orientação da amamentação é muito importante, elas se divertem, liberam um pouco as suas angústias. (Med4 UBS2)

O trabalho com grupos na atenção básica é uma alternativa para as práticas assistenciais, configurando-se em um recurso que favorece 0 aprimoramento dos envolvidos tanto no aspecto pessoal quanto no profissional. Tal atividade valoriza os diversos saberes e possibilita a intervenção criativa no processo de saúde-doença de cada indivíduo ${ }^{14}$.

A prática de atividades com grupos é responsável pela promoção e construção coletiva do conhecimento. Além disso, possibilita a oportunidade de reflexão sobre a realidade experienciada pelos participantes, favorece a ruptura da relação verticalizada entre profissional-usuário, além de facilitar a expressão de sentimentos, dúvidas e emoções ${ }^{15}$. 
Ademais, o processo educativo com gestantes, desenvolvido no âmbito grupal fortalece a autonomia e o protagonismo das mulheres e familiares no processo de nascimento. 0 conhecimento proporcionado nos grupos os capacita para fazerem escolhas na gravidez, no parto, no nascimento e no pós-parto ${ }^{11}$.

A seguir, os profissionais foram questionados quanto aos benefícios do trabalho com grupos. Por meio dos discursos, percebeu-se que eles reconheceram o grupo como uma prática positiva. Porém, a fala de Med1 UBS1 permite evidenciar que, na UBS1, embora os profissionais destacassem que no grupo as dúvidas eram esclarecidas e orientações realizadas, a atividade era realizada por acadêmicas, como complementação do trabalho dos profissionais.

$\mathrm{Na}$ realidade, o que eu vejo, é que, por exemplo, quando eu atendo sempre pergunto se elas têm dúvidas, muitas vezes, elas me dizem assim: "ah, doutora, até ali no grupo as meninas já me explicaram e tal". Então, eu acho isso uma coisa maravilhosa. Por isso, que eu disse que complementa o nosso trabalho, porque na verdade, muitas vezes as acadêmicas de enfermagem esclarecem coisas, que as gestantes não precisam nem perguntar para mim. (Med1 UBS1)

Eu acho que melhora, dá apoio, acho que dá suporte psicológico, mesmo que não tenha o profissional específico da psicologia, da saúde mental. É um momento de troca, onde ela se sente mais segura, tendo os profissionais da saúde para conversar, acho que isso é importante. (Den1 UBS1)

Eu acho que elas trocam mais ideias entre si, recebem mais informação, porque na consulta individual, se faz várias, o próprio procedimento da consulta, às vezes, não permite que tu fale assuntos, como os cuidados com bebê, alimentação, tudo que seria importante. Então, isso é uma coisa que fica, que valoriza bastante, que te evidencia bastante, e até, às vezes, dúvidas que elas não tinham antes, na troca de ideias, elas passam também ter e ter também uma resposta para aquela dúvida. (Enf1 UBS1)

A partir dos depoimentos, compreendeu-se que a realização de atividades educativas em todo ciclo gravídico e puerperal são importantes. No entanto, é durante o pré-natal que a mulher precisa ser orientada, possibilitando-lhe viver a gestação e o puerpério de maneira positiva e esclarecida, o que pode promover menos riscos de complicações e maior êxito na amamentação.

Desta forma, o vínculo entre profissional de saúde e a gestante é fundamental no serviço de saúde, visto que propicia a mulher o exercício do seu papel de cidadã, conferindo-a autonomia no que diz respeito à saúde, direitos de fala, argumentação e escolha respeitada, permitindo ao profissional conhecer a mesma para que colabore com a integração deste na manutenção de sua saúde e redução dos agravos do ciclo gravídico-puerperal ${ }^{16}$.

Frente ao exposto, entende-se que, apesar das dificuldades relatadas para a realização de grupo de gestantes, os profissionais precisam focar seus esforços no intuito de superarem os obstáculos, buscando aprender com experiências anteriores, tenham sido elas positivas ou negativas. É necessário que, além da identificação dos entraves, haja um real comprometimento dos profissionais também na elaboração de soluções.

\section{Influência do processo de formação dos profissionais de saúde para o trabalho com grupos de gestantes na UBS}

No Brasil, até a década de 1970, o modelo assistencial de saúde vigente era o médico assistencial privatista, baseado na medicina curativa e hospitalocêntrica. No contexto da Reforma Sanitária, destaca-se a VIII Conferência Nacional de Saúde que colocou em discussão a saúde como direito de todos e reformulação do Sistema Nacional de Saúde ${ }^{17}$.

Sequencialmente, em 1988, a Constituição Federal deu origem ao Sistema Único de Saúde (SUS) baseado nos princípios da universalidade, integralidade, equidade e participação social. Posteriormente, foi instituída a Lei Orgânica da Saúde 8.080/90 que regulamentou as condições para a promoção, proteção e recuperação da saúde ${ }^{18}$.

Nesta conjuntura ocorreram mudanças na assistência à saúde. Ao mesmo tempo as instituições de ensino superior vêm sofrendo expressivas modificações no sentido de alterarem suas práticas pedagógicas e implementarem mudanças na formação em saúde com objetivo de formar profissionais comprometidos com novo modelo assistencial, com olhar direcionado para o cuidado integral e humanístico ${ }^{19}$. 
No que tange aos métodos educativos, a abordagem por meio de grupos destaca-se enquanto atividade educativa coletiva. As técnicas grupais revelam-se como importante ferramenta de trabalho educativo com sujeitos coletivos, principalmente àquelas com perspectiva pedagógica, participativa e dialógica ${ }^{20}$.

No presente estudo, os profissionais de saúde foram questionados quanto á participação dos mesmo enquanto acadêmicos em grupos de gestantes. Bem como o fornecimento de conhecimento a cerca da metodologia grupal na formação acadêmica dos mesmos.

Nos postos de saúde, fazíamos não como o projeto de extensão que temos das Acadêmicas de Enfermagem. Naquela época, a coisa não era tanto assim, como tem hoje em dia. Mas trabalhamos bastante com grupo, em atividades e tal. (Enf1 UBS1)

Só atendimento, dentro da medicina, no ambulatório. Tu atendias no ambulatório, e fazia atendimento às gestantes. Eu me formei há muito tempo, não era de grupos. Não tínhamos esta formação de grupos, me formei em oitenta. (Med2 UBS2)

De nada, grupo de nada durante a graduação. (Enf2 UBS2)

Não tive essa experiência. (Enf1 UBS2)

Ao refletir sobre os depoimentos, visualiza-se que os profissionais não tiveram suporte teórico-prático durante a formação acadêmica para o desenvolvimento da prática educativa com grupos de gestantes. Além destes aspectos, a fala de Med2 UBS2 revelou uma formação voltada para ações verticalizadas com ênfase no modelo biomédico.

Observou-se que a ausência de práticas educativas, durante a formação dos participantes do estudo, refletiu na dificuldade de trabalhar com grupos em sua prática profissional. Nesta perspectiva, percebeu-se a importância do estímulo e da vivência do trabalho com grupos ainda na graduação, uma vez que o SUS requer profissionais generalistas, críticos e reflexivos capazes de atuarem nos diferentes cenários da saúde.

Conforme os depoimentos de Enf1 UBS1 e Med1 UBS1, na UBS, o grupo de gestantes era desenvolvido pelos acadêmicos. Evidenciando-se a falta de envolvimento de outros profissionais nesta atividade.

Aqui na unidade quem trabalha são as gurias (acadêmicas de enfermagem). A gente faz 0 atendimento da gestante, não o grupo. (Enf1 UBS1)

Porque quem faz o grupo de gestante são as acadêmicas de enfermagem. Nem eu, nem a enfermeira. Depois que as gestantes terminam o grupo, elas vem ser atendidas por nós (médica e enfermeira). (Med1 UBS1)

Os resultados desta pesquisa reforçam os achados de um estudo realizado no Paraná quando aponta que os profissionais de saúde habituados a trabalhar de maneira essencialmente assistencialista, enfrentam um novo desafio frente a sua atuação com as gestantes. Estes precisam buscar a capacitação profissional constantemente para desempenhar um bom papel em grupos ${ }^{21}$.

No entanto, entende-se que não é só o conhecimento que deve pautar a metodologia grupal, pois a convivência em tal espaço promove a formação de uma rede de solidariedade e de apoio que propicia a transformação de experiências individuais em coletivas. Neste sentido, é de suma importância também a formação de vínculos entre os participantes do grupo e os profissionais de saúde 22 .

Diante desta perspectiva, a atividade com grupo de gestantes constituiu-se como importante recurso para troca de experiências e conhecimentos.

A gente fazia grupos, conseguíamos juntar mais gestantes, até por causa do horário. Aqui fazemos muito cedo, muitas vezes, os profissionais ainda estão muito envolvidos com o atendimento clínico, isso é um problema nosso. (Den1 UBS1)

Eu gostei. Também vieram os acadêmicos da Universidade Federal, foi bom. Elas trocaram conhecimento, trocavam experiência, ensinaram a dar banho. Teve um que explicou como era a concepção, como o nenê se formava, foi legal. (Enf1 UBS2) 
Para mim, era bem interessante. Na verdade, era sempre, tinha dia certo e era um grupo de sala de espera. Elas chegavam todas e enquanto umas iam sendo atendidas, as outras ficavam tendo palestras, sobre várias coisas, amamentação, sobre vários tipos de doença que poderiam ter, sobre prevenção. (Med3 UBS2)

Conforme descritos nos relatos, alguns dos profissionais vivenciaram a experiência de grupo de gestantes de modo satisfatório. Percebe-se que os mesmos relacionaram suas práticas com a participação ativa das usuárias, contribuindo para a construção de saber coletivo por meio de questionamentos entre profissionais-usuários.

No entanto, a fala de Med3 UBS2 permite identificar que o profissional desenvolvia suas atividades sob o prisma tecnicista. Uma vez que traz o entendimento do grupo como palestra, no qual as mulheres permaneciam sentadas e os profissionais discorriam sobre determinados assuntos.

Em contraponto ao modelo tecnicista, a prática grupal permite 0 aprender e o ensinar em um compartilhamento mútuo. Neste processo, é fundamental que o profissional mediador participe da ação de repensar a construção do conhecimento. Com isso, ele aprende com as discussões e com os conhecimentos dos outros profissionais ${ }^{23}$.

As vivências no grupo são essenciais para o crescimento profissional e para o conhecimento das gestantes. Os debates gerados a partir de conhecimentos prévios e as expectativas das gestantes contribuem para que elas se sintam mais seguras ${ }^{24}$.

Diante do apresentado, compreende-se que a falta de fundamentação teórico-prática durante a formação acadêmica parece ter influência nas práticas atuais dos profissionais de saúde deste estudo, pois embora os profissionais reconheçam o grupo de gestante como atividade fundamental não sentem-se estimulados a mudar sua prática profissional. Tendo em vista, que os profissionais da UBS1 não se encontraram envolvidos com o grupo de gestantes e na UBS2 não ocorria a realização desta prática.

Ressalta-se que, mesmo não vivenciando a prática com grupo durante a formação acadêmica entende-se que os conhecimentos proporcionados ao longo da formação dos profissionais poderiam viabilizar o desenvolvimento de atividades com grupos. Pois o que precisa-se para o trabalho em grupos é o conhecimento prévio sobre 0 assunto e a vontade de sair da zona de conforto da assistência fundamentada na pratica medicalizada.

Além disso, é necessário que o profissional seja dinâmico, motivado a aprender. Uma possibilidade para atualização de conhecimentos é a participação/interação com projetos de extensão desenvolvidos no local de trabalho destes profissionais.

\section{Considerações Finais}

No presente estudo, os profissionais de saúde da Atenção Básica identificaram o trabalho com grupos de gestantes como uma atividade importante na atenção ao pré-natal, parto e puerpério. Eles destacaram o grupo como espaço de troca de conhecimentos e de experiências entre participantes e os profissionais e um cenário que proporciona as mulheres e seus familiares o esclarecimento das dúvidas, incentivo a autonomia na tomada de decisões referentes ao seu processo de gestação, parto e nascimento de seu filho, ao mesmo tempo possibilita a troca de saberes e práticas entre as participantes e coordenadores.

Neste estudo, os participantes relataram como dificuldades para a realização de atividades com grupo de gestante as experiências anteriores negativas, excesso de trabalho e baixa adesão das usuárias as atividades propostas.

Neste sentido, é importante que os profissionais busquem conhecer quais são as expectativas das gestantes sobre o trabalho com grupo. Além disso, propiciar a participação ativa das mulheres em todos os momentos da atividade, desde a listagem dos conteúdos que serão desenvolvidos até o modo como deverão ser trabalhados pode ser, também, uma possibilidade de sucesso nesta atividade.

O estudo também destacou que a maioria dos profissionais de saúde não vivenciou durante a sua formação 0 trabalho com grupos de gestantes. Aqueles que realizaram atividades com grupos, o fizeram ancorado no modelo biomédico, fundamentado em ações verticalizadas, nas quais o profissional com caráter tecnicista discorria sobre assuntos, enquanto as mulheres assistiam passivamente.

Os resultados encontrados neste estudo apontam lacunas que poderão ser trabalhadas para melhoria dos serviços, no intuito de promover o cuidado integral e humanístico. Sendo que a atividade com grupos de gestante uma ferramenta importante de atenção à gestante, família e comunidade.

Recomenda-se que novos estudos sejam realizados, aprofundando os aspectos que envolvam a baixa adesão das usuárias ao grupo de gestantes. Além deste aspecto, sugere-se estudos ligados ao papel dos profissionais de saúde neste cenário de atuação. 


\section{Referências}

1. Costa ES, Pinon GMB, Costa TS, Santos RCA, Souza LB. Alterações fisiológicas na percepção de mulheres durante a gestação. Rev. Rene. 2010 abr/jun;11(2):86-93.

2. Pinheiro BC, Bittar, CML. Expectativas, percepções e experiências sobre o parto normal: relato de um grupo de mulheres. Fractal, Rev. Psicol. 2013;25(3): 585-602.

3. Barbato RG, Corrêa AK, Souza MCBM. Aprender em grupo: experiência de estudantes de enfermagem e implicações para a formação profissional. Esc Anna Nery Rev Enferm. 2010 jan/mar;14(1):48-55.

4. Matos GC, Santos CC, Escobal APL, Soares MC, Meincje SMK. Grupos de gestantes: espaço para promoção do cuidado integral. Rev Enferm UFPE [internet] 2015 Mai[acesso em 2015 Set 6];9(5): 27-33. Disponível em: http://www. revista.ufpe.br/revistaenfermagem/index.php/revista/article/view/6682/pdf_7772 doi: 10.5205/reuol.6121-57155-1ED.0905201506.

5. Picinini CA, Carvalho, FT, Ourique LR, Lopes RS. Percepções e sentimentos de gestantes sobre o pré-natal. Psic.: Teor. e Pesq. 2012 jan-mar;28(1):27-33.

6. Figueiredo JV, Freitas LV, Lima TM, Oliveira AS, Damasceno AKC. Promovendo a autoridade e o poder da gestante: uma atividade da enfermagem na construção da cidadania*. Enfermagem em Foco. 2010; 1(3):124-128.

7. Minayo MCS. O desafio do conhecimento: pesquisa qualitativa em saúde. 13 ed. São Paulo: Hucitec; 2013. 407p.

8. Ministério da Saúde (BR). Conselho Nacional de Saúde. Resolução 466 de 12 de dezembro de 2012: Dispõe sobre diretrizes e normas regulamentadora de pesquisa em seres humanos. [internet] 2012 [acesso em 2015 Mar 20]. Disponível em: http://conselho.saude.gov.br/resolucoes/2012/Reso466.pdf.

9. Souza AXA, Nóbrega SM, Coutinho MPL. Representações sociais de adolescentes grávidas sobre a gravidez na adolescência. Psicologia \& Sociedade. 2012;24(3):588-96.

10. Maçola L, Vale IN, Carmona EV. Avaliação da autoestima de gestantes com uso da Escala de Autoestima de Rosenberg. Rev Esc Enferm. [internet]. 2010 [acesso em 2015 fev 6]; 44(3): 570-77. Disponível em: http://www.scielo.br/ pdf/reeusp/v44n3/04.pdf doi: 10.1590/S0080-62342010000300004.

11. Zampieri MFM, Gregório VRP, Custódio ZAO, Regis MI, Brasil C. Processo educativo com gestantes e casais grávidos: possibilidade para transformação e reflexão da realidade. Texto Contexto Enferm. 2010;19(4):719-27.

12. Andrade ACV, Schwalm MT, Ceretta LB, Dagostin VS, Soratto MT. Planejamento das ações educativas pela equipe multiprofissional da Estratégia Saúde da Família. O Mundo da Saúde. 2013;37(4):439-49.

13. Franco TAV, Silva JLL, Daher DV. Educação em saúde e a pedagogia dialógica: uma reflexão sobre grupos educativos na atenção básica. Informe-se em promoção da saúde. 2011;7(2):19-22.

14. Silva MZN, Andrade AB, Bosi MLM. Acesso e acolhimento no cuidado pré-natal à luz de experiências de gestantes na Atenção Básica. Saúde Debate. 2014 out-dez;38(103):805-16.

15. Diaz C.M.G, Hoffmann IC, Costenaro RGS, Soares RS, Silva BR, Laval BC. Vivências educativas da equipe de saúde em unidade gineco-obstetrícia. Cogitare Enferm.[internet] 2010 abr/ jun [acesso em Mar 18]; 15(2):364-367. Disponíel em: http://ojs.c3sl.ufpr.br/ojs/index.php/cogitare/article/view/17879/11668 doi: http://dx.doi.org/10.5380/ce.v15i2.17879

16. Gryschek ALFPL, Nichiata, LYI, Fracolli LA, Oliveira MAF, Pinho PH. Tecendo a rede de atenção à saúde da mulher em direção à construção da linha de cuidado da gestante e puérpera, no Colegiado de Gestão Regional do Alto Capivari - São Paulo. Saúde Soc. São Paulo. 2014;23(2):689-700.

17. Souza BC, Bernardo ARC. O Papel do Enfermeiro no Pré-Natal Realizado no Programa de Saúde da Família-PSF. Interfaces Científicas. Saúde e Ambiente. 2013 out;:2(1):83-94.

18. Brasil. Ministério da saúde. Lei n.8.080 de 19 de setembro de 1990. Dispõem sobre as condições para a promoção, proteção e recuperação da saúde. Diário Oficial da União, Brasilia, 19 set. 1990. Seção1, p.18055.

19. Dias ES, Lima LD, Teixeira M. A trajetória da política nacional de reorientação da formação profissional em saúde no SUS. Revista ciência \& saúde coletiva. 2013;18(6):1613-24.

20. Alves GG, Aerts D. As práticas educativas em saúde e a Estratégia Saúde da Família. Ciência \& Saúde Coletiva. 2011;16(1):319-25.

21. Souza VB, Roecker S, Marcon SS. Ações educativas durante a assistência pré-natal: percepção de gestantes atendidas na rede básica de Maringá-PR. Rev. Eletr. Enf [Internet] 2011 abr/jun [acesso em Mai 9];13(2):199-210. Disponível em: https://www.fen.ufg.br/fen_revista/v13/n2/v13n2a06.htm doi:http://dx.doi.org/10.5216/ree.v13i2.10162. 
22. Silva ALS, Nascimento ER, Coelho EAC, Nunes IM. Atividades educativas no pré-natal sob o olhar de mulheres grávidas. Revista cubana de Enfermagem. 2014;30(1):1-10.

23. Borges TS, Alencar G. Metodologias ativas na promoção da formação crítica do estudante: 0 uso das metodologias ativas como recurso didático na formação crítica do estudante do ensino superior. Cairu em Revista. Jul/Ago 2014;03(04):19-143.

24. Frigo LF, Silva RM, Boeira GS, Manfio F. A importância dos grupos de gestante na atenção primária: um relato de experiência. Rev Epidemiol Control Infect. 2012;2(3):113-4.

\section{Joice Ane Teixeira}

Endereço para correspondência - Rua Gomes Carneiro, $n^{\circ} 1$, Bairro: Centro, CEP: 96010-610, Pelotas, RS, Brasil.

E-mail: anetxra@gmail.com

Lattes: http://lattes.cnpq.br/4764437914103104

Marilu Correa Soares - enfmari@uol.com.br

Ana Paula de Lima Escobal - anapaulaescobal@hotmail.com

Kamila Dias Gonçalves - kamila_goncalves_@hotmail.com

Greice Carvalho de Matos - greicematos1709@hotmail.com

Bruna Madruga Pires da Silva - brunamadrugapires@hotmail.com

Katia da Silva Rocha - katiadasilvarocha@hotmail.com

Enviado em 28 de maio de 2016. Aceito em 06 de agosto de 2016. 